\title{
Nanoemulsions for the Encapsulation of Hydrophobic Actives
}

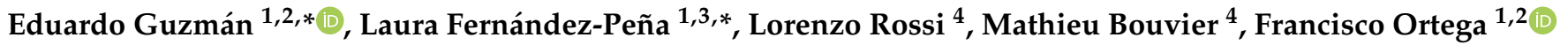 \\ and Ramón G. Rubio $1,2, *$ (D) \\ 1 Departamento de Química Física, Facultad de Ciencias, Universidad Complutense de Madrid, \\ Ciudad Universitaria s/n, 28040 Madrid, Spain; fortega@quim.ucm.es \\ 2 Instituto Pluridisciplinar, Universidad Complutense de Madrid, Paseo Juan XXIII, 28040 Madrid, Spain \\ 3 Centro de Espectroscopía y Correlación, Universidad Complutense de Madrid, Ciudad Universitaria s/n, \\ 28040 Madrid, Spain \\ 4 École Nationale Supérieure de Chimie de Lille (ENSCL), Avenue Mendeleiev, \\ 59652 Villeneuve-d'Ascq, France; lrossi@enscl.fr (L.R.); mathieu.bouvier@enscl.fr (M.B.) \\ * Correspondence: eduardogs@quim.ucm.es (E.G.); laura.fernandez.pena@ucm.es (L.F.-P.); \\ rgrubio@quim.ucm.es (R.G.R.); Tel.: +34-91-3944107 (E.G.); +34-91-3944263 (L.F.-P.); +34-91-3944123 (R.G.R.)
}

Citation: Guzmán, E.;

Fernández-Peña, L.; Rossi, L.; Bouvier, M.; Ortega, F.; Rubio, R.G.

Nanoemulsions for the Encapsulation of Hydrophobic Actives. Cosmetics 2021, 8, 45. https://doi.org/10.3390/ cosmetics 8020045

Academic Editor: Enzo Berardesca

Received: 6 May 2021

Accepted: 31 May 2021

Published: 1 June 2021

Publisher's Note: MDPI stays neutral with regard to jurisdictional claims in published maps and institutional affiliations.

Copyright: (c) 2021 by the authors. Licensee MDPI, Basel, Switzerland. This article is an open access article distributed under the terms and conditions of the Creative Commons Attribution (CC BY) license (https:// creativecommons.org/licenses/by/ $4.0 /)$.

\begin{abstract}
This work analyzes the dispersion of two highly hydrophobic actives, (9Z)-N-(1,3dihydroxyoctadecan-2-yl)octadec-9-enamide (ceramidelike molecule) and 2,6-diamino-4-(piperidin1-yl)pyrimidine 1-oxide (minoxidil), using oil-in-water nanoemulsions with the aim of preparing stable and safe aqueous-based formulations that can be exploited for enhancing the penetration of active compounds through cosmetic substrates. Stable nanoemulsions with a droplet size in the nanometric range (around $200 \mathrm{~nm}$ ) and a negative surface charge were prepared. It was possible to prepare formulations containing up to $2 \mathrm{w} / \mathrm{w} \%$ of ceramide-like molecules and more than $10 \mathrm{w} / \mathrm{w} \%$ of minoxidil incorporated within the oil droplets. This emulsions evidenced a good long-term stability, without any apparent modification for several weeks. Despite the fact that this work is limited to optimize the incorporation of the actives within the nanoemulsion-like formulations, it demonstrated that nanoemulsions should be considered as a very promising tool for enhancing the distribution and availability of hydrophobic molecules with technological interest.
\end{abstract}

Keywords: encapsulation; oil-in-water nanoemulsions; hydrophobic substance; ceramide; minoxidil

\section{Introduction}

Colloidal carriers are good candidates for the encapsulation of hydrophobic actives, enhancing their availability [1-4]. The encapsulation procedures are based on the entrapping of the molecules within the carrier material, which results in the formation of particulate systems, favoring the dispersion and distribution of the actives in an aqueous environment $[5,6]$. Furthermore, the design of appropriate platforms for the solubilization and encapsulation of specific molecules needs to take into account several aspects related to the protection of the encapsulated compounds against degradation, and the long-term stability of the obtained formulations [7-10].

In the last two decades, oil-in-water nanoemulsions, with suitable compositions and physico-chemical properties, have been exploited for the encapsulation and delivery of different active molecules with interest in different industries, mainly the biomedical, food and cosmetic industries [11-13]. The growing interest in the use nanoemulsions for the loading and release of cosmetic actives is related to their recognized ability for facilitating the penetration of the molecules through cosmetic substrates, i.e., hair fibers or skin [14-17]. This has stimulated their application for enhancing the applicability of many actives, which presents poor stability and limited solubility in water [18].

This work explores the use of oil-in-water $(\mathrm{o} / \mathrm{w})$ nanoemulsions as platforms for the dispersion of two hydrophobic molecules, namely (9Z)-N-(1,3-dihydroxyoctadecan2-yl)octadec-9-enamide (ceramide-like molecule, hereinafter CER) and 2,6-diamino-4- 
(piperidin-1-yl)pyrimidine 1-oxide (minoxidil, hereinafter MIN). The first one is a synthetic analogous of the main ceramide appearing in humans, which plays an essential role in the control of skin hydration. The disruption and degradation of the ceramide layer have a critical impact on the aging of skin and the emergence of wrinkles. These unsightly effects are associated with the modification of the hydration balance across the epidermal barrier. Therefore, it is expected that cosmetic formulations containing an exogenous source of a ceramide-like molecule may present a repairing effect for skin by hindering, at least partially, the undesirable effects associated with aging [19]. The second compound is a beta-blocker, which is frequently used in both medical products and cosmetic formulations for the prevention and treatment of alopecia due to its recognized role on the stimulation of the circulatory flow within the hair follicles [20,21]. Despite the fact that the real mechanism underlying the performance of the above molecules is almost unknown, different studies have evidenced their effectiveness, which has fostered many research efforts trying to optimize commercial formulations containing such molecules. However, the difficulties associated with the encapsulation process of both compounds lead in many cases to formulations presenting a reduced amount of the active molecules, which in turn reduces their efficacies. Furthermore, such formulations are relatively expensive, which is an important drawback towards commercialization [14,22]. Therefore, the preparation of simple formulations with long-term stability that can enhance the encapsulation yield of hydrophobic molecules, and consequently their bioavailability, is a challenge for the cosmetic and pharmaceutical industries. This makes of the incorporation of CER and MIN within the oil droplets of aqueous-based emulsion-like systems a viable alternative to ensure the stabilization of the actives and contribute to their dispersion. Furthermore, this type of formulation may result in more convenient application because their liquid nature makes easy their spreading onto the cosmetic substrate, and their high aqueous content may facilitate the removal of the residues upon rinsing. This work tries to exploit the phase inversion induced by the temperature method (PIT) for preparing oil-in-water nanoemulsions loaded with hydrophobic actives. The PIT method is categorized as a low energy method for the preparation of nanoemulsions, allowing the preparation of oil-in-water systems from a precursor water-in-oil dispersion taking advantage of the ability of the emulsifying agent for changing its relative solubility within the oily and aqueous phase with the change of temperature. Thus, the PIT method offers a tunable way for preparing nanoemulsions, which allows the prevention of the degradation of the encapsulated compounds and consumes low amounts of energy. Furthermore, this approach may be easily scalable from laboratory to industrial applications [23].

\section{Materials and Methods}

\subsection{Materials}

Oleic acid (purity > 99\%) supplied by Sigma-Aldrich (Saint Louis, MO, USA), were used as oil phase of the different prepared nanoemulsions. Polyoxyethylene(20)sorbitan monooleate (Tween 80) purchased from Sigma-Aldrich (Saint Louis, MO, USA) was used for nanoemulsion stabilization. The two hydrophobic compounds to be encapsulated: (9Z)$\mathrm{N}$-(1,3-dihydroxyoctadecan-2-yl)octadec-9-enamide (CER) and 2,6-diamino-4-(piperidin-1yl)pyrimidine 1-oxide (MIN) were supplied by Avanti Polar Lipids, Ltd. (Alabaster, AL, USA) and Sigma Aldrich (Saint Louis Sigma, Aldrich, MO, USA), respectively. Figure 1 shows the molecular structure of both hydrophobic actives.

Ultrapure deionized water used for cleaning and solution preparation was obtained by a multicartridge purification system aquaMAX ${ }^{\mathrm{TM}}$-Ultra 370 Series (Young Lin Instrument, Co., Gyeonggi-do, Korea). The water used had a resistivity higher than $18 \mathrm{M} \Omega \cdot \mathrm{cm}$, and a total organic content lower than $6 \mathrm{ppm}$. 
<smiles>CCCCCCCC/C=C\CCCCCCCCCCCC(O)C(CO)NC(=O)CCCCCCCCCC</smiles>

(a)<smiles>Nc1cc(N2CCCCC2)nc(N)[n+]1[O-]</smiles>

(b)

Figure 1. (a) Molecular structure of (9Z)-N-(1,3-dihydroxyoctadecan-2-yl)octadec-9-enamide) and (b) 2,6-diamino-4-(piperidin-1-yl)pyrimidine 1-oxide.

\subsection{Nanoemulsion Preparation}

Nanoemulsions were prepared by weighting using an analytical balance with a precision of $\pm 0.1 \mathrm{mg}$. The preparation of the nanoemulsions was done by following phase inversion induced by temperature method (PIT) adapted from the work by Tong et al. [24]. This procedure takes advantage of the use of a non-ionic surfactant containing polyoxyethylene groups, which can change its relative solubility in water and oil phases as function of the temperature [25,26], and can be summarized as follows: initially, $4 \mathrm{~g}$ of a precursor water-in-oil emulsion equal amount of surfactant and oil phase $(30-40 \mathrm{w} / \mathrm{w} \%$ of the mixture) is prepared by adding the surfactant $(30-40 \mathrm{w} / \mathrm{w} \%)$ and a small amount of water $(10-20 \mathrm{w} / \mathrm{w} \%$ of the mixture) to the oil phase. This mixture is homogenized under magnetic stirring $(1000 \mathrm{rpm})$ to obtain the precursor water-in-oil emulsion. Then, the precursor emulsion is heated at relatively high temperature $\left(70 \pm 1^{\circ} \mathrm{C}\right)$ under continuous stirring and diluted by adding water dropwise up to obtain a dispersion with a water content about $50 \mathrm{w} / \mathrm{w} \%$. Thus, it is possible to force the inversion from the precursor water-in-oil emulsion to an oil-in-water one. After the dilution, the dispersion is maintained under continuous agitation at $70 \pm 1{ }^{\circ} \mathrm{C}$ overnight to ensure the homogenization of the dispersion, and in those case in which actives are included within the dispersion for ensuring a good solubilization and distribution of the components within the formulation. It should be noted that the heating time can be reduced without compromising the preparation procedure. However, the use of such long time allows ensuring the homogeneity of the obtained dispersions. Finally, the oil-in-water emulsion is left for cooling down at room temperature during $24 \mathrm{~h}$, and then diluted with water to obtain the oil-in-water nanoemulsion with the a final oil content in the range $10-30 \mathrm{w} / \mathrm{w} \%$ of the total formulation. During the preparation of the nanoemulsions loaded with the active molecules, these were pre-dissolved in the oil phase of the precursor water-in-oil emulsion. In particular, formulations containing the ceramide-like molecule in the range $0-2.5 \mathrm{w} / \mathrm{w} \%$ and minoxidil in the range $0-10 \mathrm{w} / \mathrm{w} \%$.

It should be noted that the choice of the temperature and time for preparation of the formulations was chosen following the results reported by Tong et al. [24]. They demonstrated that oil-in-water emulsions remaining without any signature of phase separation at high temperature during a period of at least $12 \mathrm{~h}$ will maintain such stability upon their cooling down and subsequent dilution. Furthermore, the temperature used for the preparation process is well below of the melting point of the encapsulated actives, and hence the induction of any thermal degradation of the formulations upon heating cannot be expected.

\subsection{Characterization of the Nanoemulsions}

Nanoemulsions were characterized by electrophoretic mobility measurements and Dynamic Light Scattering (DLS) using a Nanosizer ZS (Malvern instruments, Malvern, UK) [5,27-33].

The effective charge density of the droplets dispersed in the aqueous phase may be inferred from measurements of the electrophoretic mobility, $u_{e}$, obtained using Laser Doppler 
velocimetry. The electrophoretic mobility is directly proportional to the zeta potential, $\zeta$, which gives a measurement of the effective charge of the droplets, by Henry's equation [34]

$$
u_{e}=\frac{2}{3 \eta} \varepsilon \zeta f(\kappa a)
$$

where $\varepsilon$ and $\eta$ represent the dielectric permittivity and the viscosity of the continuous phase, respectively, and $f(\kappa a)$ is the Henry function, which for particles big enough assumes a value of 1.5 (Smoluchowski approach) [35].

DLS measurements were performed at $25^{\circ} \mathrm{C}$ in a quasi-backscattering configuration (scattering angle, $\theta=173^{\circ}$ ) using a He-Ne laser (wavelength, $\lambda=632 \mathrm{~nm}$ ). DLS experiments allow one to obtain the time dependence of the normalized intensity autocorrelation function, $g^{(2)}(q, t)$ that for a dispersion of monodisperse scatters presenting Brownian motion, can be described in terms of an single exponential decay [36]

$$
g^{(2)}(q, t)-1=\beta e^{-2 t / \tau},
$$

where $t$ and $\tau$ are the time and the mean relaxation time, respectively, and $q=(4 \pi n / \lambda) \sin$ $(\theta / 2)$ is the wave vector, with $n$ being the continuous phase refractive index $(n=1.33)$. In Equation (1), $\beta$ is an optical coherence factor, which is generally found to be close to 1 . The analysis of the intensity autocorrelation functions allows one to estimate the apparent diffusion coefficient $D_{a p p}=1 / \tau q^{2}$ that for spherical scatters diffusing in a continuous Newtonian medium allows one to estimate the size of the droplets as the apparent hydrodynamic diameter $d_{a p p}^{h}$ ) by the Stoke-Einstein relationship

$$
d_{a p p}^{h}=\frac{k_{B} T}{3 \pi \eta D_{a p p}}
$$

where $k_{B}$ and $T$ are referred to the Boltzmann constant and the absolute temperature, respectively.

\subsection{Analysis of the Stability of the Formulations}

The stability of the emulsions was evaluated by the change of the average size defined in terms of the changes of the apparent hydrodynamic diameter upon aging of the formulations at room temperature (in the range $20-25^{\circ} \mathrm{C}$ ). Furthermore, visual assessing of the stability was also performed by the emergence of gravitational destabilization process that results in the appearance of phase separation (frequently an oil layer on the top of the formulation).

\section{Results}

\subsection{Characterization of Bare Oleic Acid in Water Nanoemulsions}

A preliminary step towards the use of mixtures of water, oleic acid and Tween 80 as platforms for the encapsulation of active molecules is seeking the most suitable composition for preparing stable nanoemulsions. This makes it necessary to study dispersions with different compositions, i.e., different contents of oil (oleic acid), water and surfactant (Tween 80). Table 1 summarizes the screened compositions for determining the most suitable compositions for the preparation stable nanoemulsions which can later be used for the encapsulation of active molecules.

The only compositions which enable the preparation of stable formulations are those containing the same weight fraction of surfactant (Tween 80) and oil phase (oleic acid), with the increase in the oil concentration in relation to that of Tween 80 resulting in the destabilization of the dispersion. Thus, phase separation was observed for dispersions containing $75 \mathrm{w} / \mathrm{w} \%$ of water after 20 days when the concentration of oil is 1.5 times than of the Tween 80 , whereas the stability window drops down to 8 days for dispersions in which the oil:surfactant ratio is increased up to 2 . On the other side, nanoemulsions with an 
oil:surfactant ratio of 1 remain stable after 6 months of aging. Therefore, it is clear that the concentration of surfactant must be at least equal to that of the oil phase in the dispersion, for ensuring the complete dispersion of the oil phase as droplets within the aqueous phase, avoiding any gravitational destabilization phenomena. This can be understood considering that the stabilization of nanoemulsions requires the formation of a dense surfactant shell around the oil droplets to prevent the coalescence and Ostwald ripening. These phenomena lead to the formation of droplet aggregates, which can separate from the emulsion.

Table 1. Summary of the compositions of the different nanoemulsions tested, and their stability.

\begin{tabular}{ccccc}
\hline $\begin{array}{c}\text { Water } \\
(\mathbf{w} / \mathbf{w} \mathbf{)}\end{array}$ & $\begin{array}{c}\text { Tween } \mathbf{8 0} \\
\mathbf{( w / w \% )}\end{array}$ & $\begin{array}{c}\text { Oleic Acid } \\
\mathbf{( w / w} \%)\end{array}$ & Oleic Acid/Tween 80 Ratio & Stability \\
\hline 50.00 & 25.00 & 25.00 & 1.00 & Yes \\
50.00 & 16.67 & 33.33 & 2.00 & No \\
60.00 & 20.00 & 20.00 & 1.00 & Yes \\
60.00 & 10.00 & 30.00 & 2.00 & No \\
68.00 & 16.00 & 16.00 & 1.00 & Yes \\
68.00 & 10.66 & 21.33 & 2.00 & No \\
70.00 & 15.00 & 15.00 & 1.00 & Yes \\
70.00 & 10.00 & 20.00 & 2.00 & No \\
75.00 & 12.50 & 12.50 & 1.00 & Yes \\
75.00 & 10.00 & 15.00 & 1.50 & No \\
75.00 & 8.25 & 16.75 & 2.00 & No \\
80.00 & 10.00 & 10.00 & 1.00 & Yes \\
\hline
\end{tabular}

Moreover, the use of Tween 80 for the stabilization of the oleic acid droplets allows preparing formulations with a high water content, in the range of 50-80 w/w\%, appearing phase separations for dispersion containing higher water content. However, the emulsions including water in the range 50-68 w/ $\mathrm{w} \%$ appear very viscous, and should be discarded for encapsulation purposes due to the difficulties associated with their practical use. Therefore, nanoemulsions with a water content in the range $68-80 \mathrm{w} / \mathrm{w} \%$ and an oil:surfactant ratio of 1 appears as the most suitable alternative for the encapsulation of hydrophobic actives within the oil droplets when dispersions containing water, Tween 80 and oleic acid are considered.

The characterization of the stable nanoemulsions was performed in terms of the droplet dimensions evaluated in terms of the apparent hydrodynamic diameter obtained from DLS measurements and the effective surface charge of the droplets evaluated in terms of the electrophoretic mobility (see Equation (1)). This latter parameter provides information on the potential stability of the formulations. Thus, high absolute values of the mobility, or zeta potential, are indicatives of a high surface charge of the droplets, which provides to the droplets with a high resistance against the aggregation. Figure 2 shows the autocorrelation functions of intensities and the respective intensity distributions obtained from the analysis of three stable nanoemulsions contained fixed water contents $(50,60$ and $70 \mathrm{w} / \mathrm{w} \%$ ), and a fixed oil:surfactant ratio of 1 . It should be noted that the high turbidity of the samples make impossible their direct measurement using DLS, and hence a dilution by a factor 10 of the nanoemulsions was required before performing the measurements. Furthermore, this also contributes to the reduction of the viscosity of the samples. It should be noted that any of the original nanoemulsions undergo destabilization upon dilution. This would be evidenced on the DLS results from the emergence of multiple contributions to the intensity distributions.

The results show that the autocorrelation functions decay over long times, appearing very similar independently of the composition of the dispersions. Furthermore, the intensity distributions obtained from the analysis of the autocorrelation functions shown in Figure $2 b$ are considerably broad and similarly independent of the nanoemulsion composition, which indicates a high polydispersity of the droplets. It should be noted that the results suggest that once stable dispersions are obtained, the increase in the content of 
water phase of the nanoemulsions does not lead to any significant change in the size of the droplets. Thus, on the basis of the above results it may be expected that an increase in the oil content can lead to an increase in the number of droplets, and hence considering that the surfactant:oil ratio remains constant, the distribution of surfactant molecules within the oil droplet/water interfaces should not be significantly modified for nanoemulsions with different weight content of water and fixed surfactant:oil ratio of 1 .
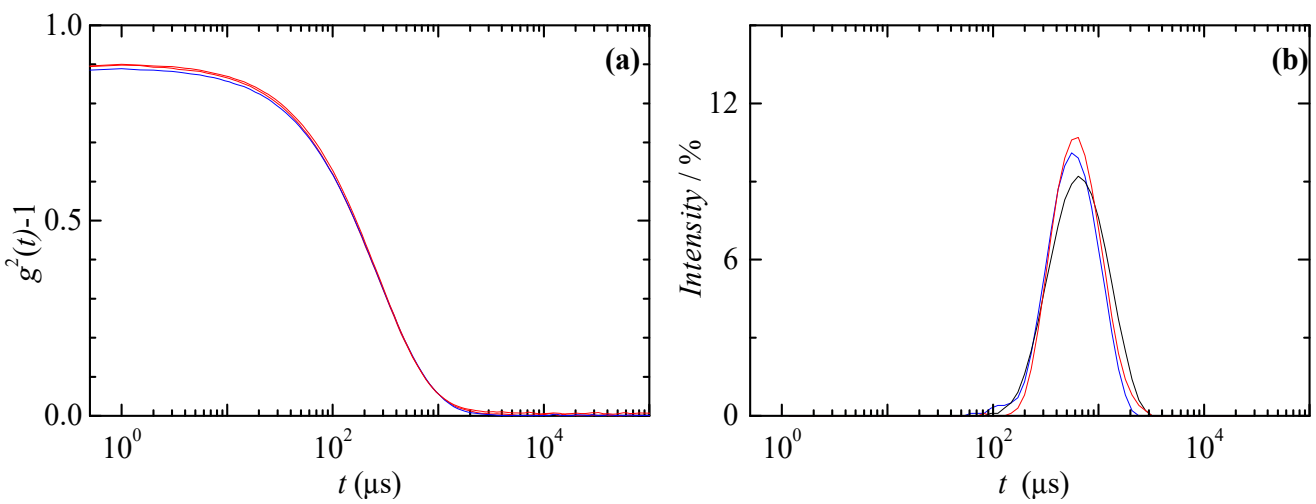

Figure 2. DLS study of different nanoemulsions with different water contents and a fixed oil:surfactant ratio of 1 . (a) Autocorrelation functions of intensities. (b) Intensity distributions. The different plots correspond to nanoemulsions with different water contents: (-) $50 \mathrm{w} / \mathrm{w} \%$, (一) $60 \mathrm{w} / \mathrm{w} \%$ and (-) $70 \mathrm{w} / \mathrm{w} \%$. Notice that the high turbidity of the mixtures makes necessary a dilution of the sample by a factor 10 for performing DLS measurements.

A more detailed analysis of the nanoemulsions may be done in terms of the apparent hydrodynamic diameter, calculated from the diffusion coefficient obtained by DLS from the intensity distribution (Figure 2b), and the electrophoretic mobility. Figure 3 shows the water content $\left(c_{\mathrm{H}_{2} \mathrm{O}}\right)$ dependences of the apparent hydrodynamic diameter and the electrophoretic mobility for nanoemulsions with an oil:surfactant ratio of 1 .
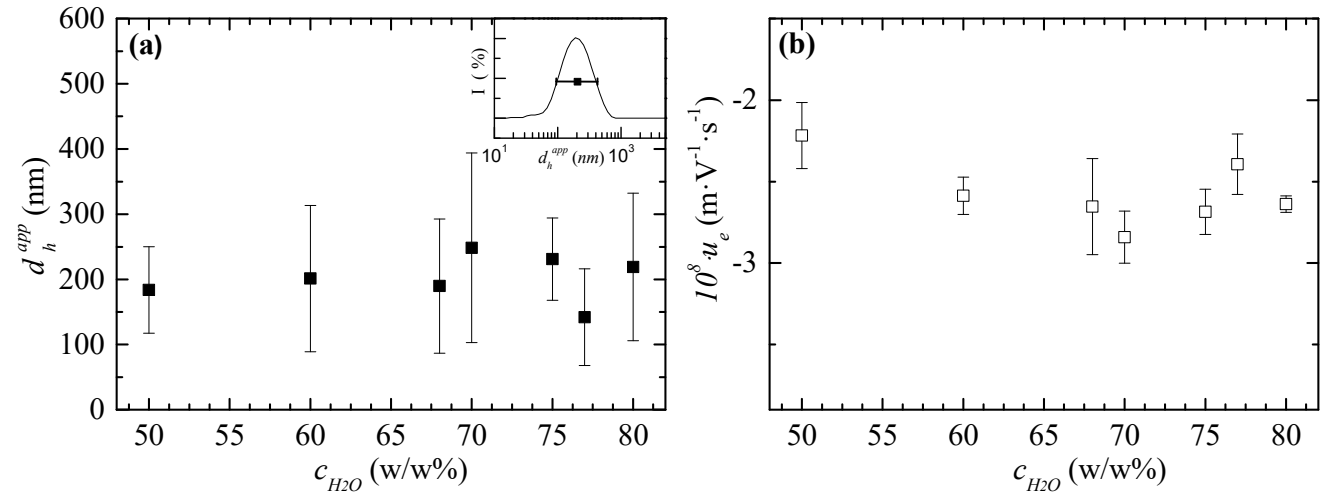

Figure 3. (a) Dependence of the apparent hydrodynamic diameter on the water concentration for nanoemulsions with a fixed oil:surfactant ratio of 1 . The error bars provide information of the width at half height of the hydrodynamic diameter distributions defined as is shown in the inset. (b) Dependence of electrophoretic mobility on the water concentration for nanoemulsions with a fixed oil:surfactant ratio of 1 . Notice that the high turbidity of the mixtures makes necessary a dilution of the sample by a factor 10 for performing DLS measurements.

The results indicate that the droplets are relatively big with an average apparent hydrodynamic diameter about $200 \mathrm{~nm}$. Furthermore, according to the negative values of the electrophoretic mobility values, the nanoemulsions should be considered as relatively stable systems due to their negative net charge evidenced on the negative values of the electrophoretic mobility reported in Figure $3 b$ (equivalent to a zeta potential of $-30 \pm 2 \mathrm{mV}$ ). 
Considering that the Tween 80 is a non-ionic surfactant, it is expected that the negative charge may arise from the incorporation of some oleic acid molecules within the interfacial layers, with this oleic acid having their dissociated heads oriented towards the water phase $[37,38]$. This results in electrostatic repulsions between the droplets, which provides stability to the formulations. Therefore, the above results suggest that the nanoemulsions studied can be suitable platforms for the encapsulation of hydrophobic actives.

\subsection{Encapsulation of CER and MIN in Oleic Acid in Water Nanoemulsions}

On the basis of the results discussed above, nanoemulsions containing $75 \mathrm{w} / \mathrm{w} \%$ of water and an oil:surfactant ratio of 1 were initially tested as potential candidates for the encapsulation of CER and MIN. This choice was selected considering that such composition corresponds to an intermediate value between the onset on the destabilization region (dispersions with a water content above $80 \mathrm{w} / \mathrm{wt} \%$ ) and the region where highly viscous nanoemulsions were obtained (dispersions with a water content below $68 \mathrm{w} / \mathrm{wt} \%$ ). The first step was possible to analyze the maximum amount of CER that may be accepted for a nanoemulsion with a specific composition without compromising the stability of the dispersion. This was possible by preparing independent formulations containing increasing amounts of the active molecule, which allows the evaluation of the maximum content that can be encapsulated from the appearance of an excess of the active substances without being solubilized in the vial or by the emergence of the instability of the formulation due to the incorporation of the active substance, i.e., induction of phase separation due to the incorporation of the active substance.

The results showed that the considered nanoemulsions with a water content of $75 \mathrm{w} / \mathrm{w} \%$ and an oil:surfactant ratio of 1 may include up to $1.5 \mathrm{w} / \mathrm{w} \%$ of the ceramide-like molecule, without any signature of phase separation or sedimentation of the encapsulated molecule. This means that it is possible to include $2.7 \times 10^{-3} \mathrm{~mol}$ of CER for each $100 \mathrm{~g}$ of the formulation. The increase in the CER content up to $1.6 \mathrm{w} / \mathrm{w} \%$ of the formulation leads to the separation of part of the ceramide-like molecule as a precipitate at the bottom of the vial containing the formulation. Figure 4 shows the dependences of the average hydrodynamic diameter and the electrophoretic mobility on the amount of ceramide-like molecule $\left(c_{C E R}\right)$ included within the nanoemulsion droplets.

The results shows that the ceramide-like molecule can be incorporated within the nanoemulsion up to a maximum concentration of $1.5 \mathrm{w} / \mathrm{w} \%$ without any significant change in the size of the droplets. However, the incorporation of increasing amounts of ceramide within the nanoemulsions leads to a broadening of the size distribution (bigger error bars). This may be associated with an increase in the polydispersity of the droplets in relation to the nanoemulsions without the incorporation active ingredient. Thus, the incorporation of the ceramide-like molecule within the nanoemulsion leads to an increase in the polydispersity index (PDI) by a factor of around 2 as is reported in the results in Table 2. The absence of a clear dependence of the half height of the size distribution, and hence of the polydispersity index, on the concentration of solubilized ceramide-like molecule appears as a signature of the absence of any significant destabilization of the nanoemulsions upon the encapsulation process. The electrophoretic mobility value of the nanoemulsion (Figure $4 \mathrm{~b}$ ) remains in negative values independently of the amount of encapsulated ceramide-like molecule. However, a slight decrease in the absolute value of the electrophoretic mobility of the nanoemulsions with the increase in the ceramide-like molecule concentration was observed. This may be rationalized considering that a part of the ceramide-like molecule is placed at the interfacial layer adsorbed at the dropletaqueous phase interface, replacing negatively charged oleic acid molecules and hence the effective charge density of the droplets is reduced. However, a detailed analysis of the zeta potential indicates that the surface charge of the droplets upon the encapsulation of the ceramide-like molecule should be considered almost negligible. This indicates that the incorporation of the ceramide-like molecules within the oil droplets does not 
significantly modify the stability of the nanoemulsions, which appears mainly governed by the electrostatic repulsion between the charged droplets.
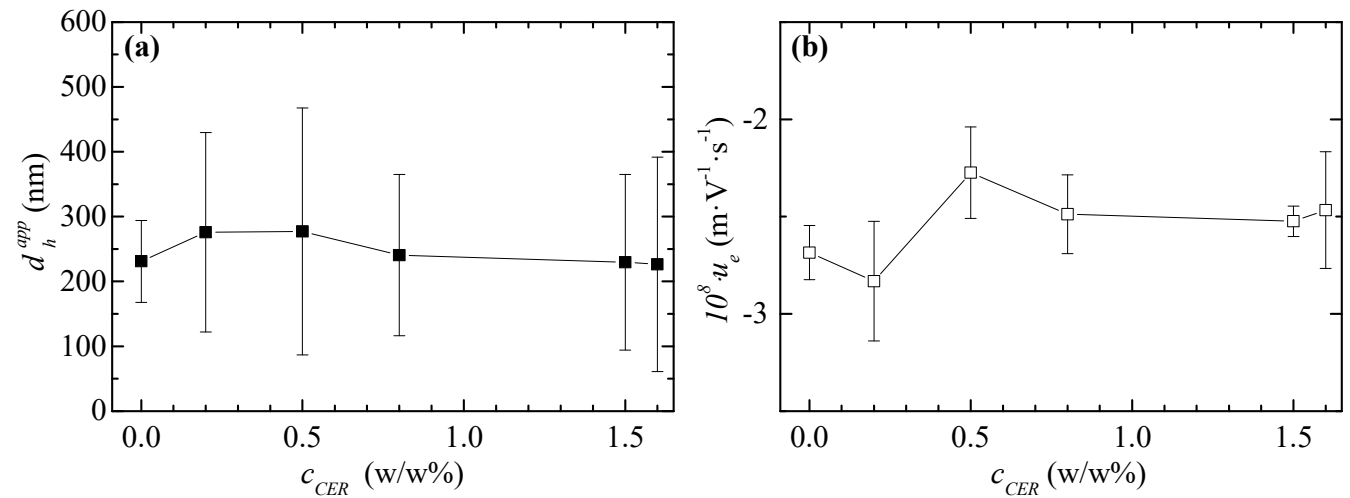

Figure 4. (a) Dependence of the apparent hydrodynamic diameter on the encapsulated CER concentration for nanoemulsions with an oil:surfactant ratio of 1 and a fixed amount of water of $75 \mathrm{w} / \mathrm{w} \%$. The error bars provide information of the width at half height of the hydrodynamic diameter distributions. (b) Dependence of electrophoretic mobility on the encapsulated CER concentration for nanoemulsion with an oil:surfactant ratio of 1 and a fixed amount of water of $75 \mathrm{w} / \mathrm{w} \%$. The lines are guides for the eyes. Notice that the high turbidity of the mixtures makes necessary a dilution of the sample by a factor 10 for performing DLS measurements.

Table 2. Average hydrodynamic diameter, polydispersity index, electrophoretic mobility and zeta potential for nanoemulsions with a water content of $75 \mathrm{w} / \mathrm{w} \%$ and a fixed oil ratio of 1 loaded with different amounts of the ceramide-like molecule.

\begin{tabular}{ccccc}
\hline $\begin{array}{c}\text { Loaded CER } \\
(\mathbf{w} / \mathbf{w} \mathbf{\%})\end{array}$ & $\begin{array}{c}\boldsymbol{d}_{\boldsymbol{h}}^{a p p} \\
(\mathbf{n m})\end{array}$ & PDI & $\begin{array}{c}\mathbf{1 0}^{\mathbf{8}} \boldsymbol{u}_{\boldsymbol{e}} \\
\left(\mathbf{m} \cdot \mathbf{V}^{-\mathbf{1}} \cdot \mathbf{s}^{-\mathbf{1}}\right)\end{array}$ & $\begin{array}{c}\text { Zeta Potential } \\
\mathbf{( m V )}\end{array}$ \\
\hline 0 & 231 & 0.137 & $-(2.7 \pm 0.1)$ & $-(30 \pm 2)$ \\
0.2 & 275 & 0.279 & $-(2.8 \pm 0.3)$ & $-(33+5)$ \\
0.5 & 277 & 0.344 & $-(2.3 \pm 0.2)$ & $-(29 \pm 3)$ \\
0.8 & 240 & 0.259 & $-(2.5 \pm 0.2)$ & $-(29 \pm 3)$ \\
1.5 & 229 & 0.295 & $-(2.5 \pm 0.1)$ & $-(32 \pm 2)$ \\
1.6 & 226 & 0.365 & $-(2.5 \pm 0.3)$ & $-(34 \pm 5)$ \\
\hline
\end{tabular}

The same approach used for loading the ceramide-like molecule within the nanoemulsions was exploited for including the second active ingredient, i.e., the minoxidil. The results showed that minoxidil can be incorporated within the nanoemulsions in a concentration that is several times higher than the corresponding one for the ceramide-like molecule. Thus, nanoemulsions with up to $10 \mathrm{w} / \mathrm{w} \%$ of minoxidil were obtained without any signature of destabilization on the formulation. This means that it was possible to include at least $4.8 \times 10^{-2} \mathrm{~mol}$ of minoxidil per each $100 \mathrm{~g}$ of dispersion. This does not mean that such concentration is the maximum amount of minoxidil that the formulation can incorporate. However, the application of formulations containing $10 \mathrm{w} / \mathrm{w} \%$ of minoxidil involves between 2 and 5 -fold the conventional dosages of most commercial formulations for topical application, and hence this work has been limited to the incorporation within the nanoemulsions of a reasonable minoxidil concentration for a practical purpose [39]. Figure 5 shows a comparison of the dependence of the droplet size of the nanoemulsion on the concentration of both active ingredients $\left(c_{\text {active }}\right)$, as well as the electrophoretic mobility of the droplets. 

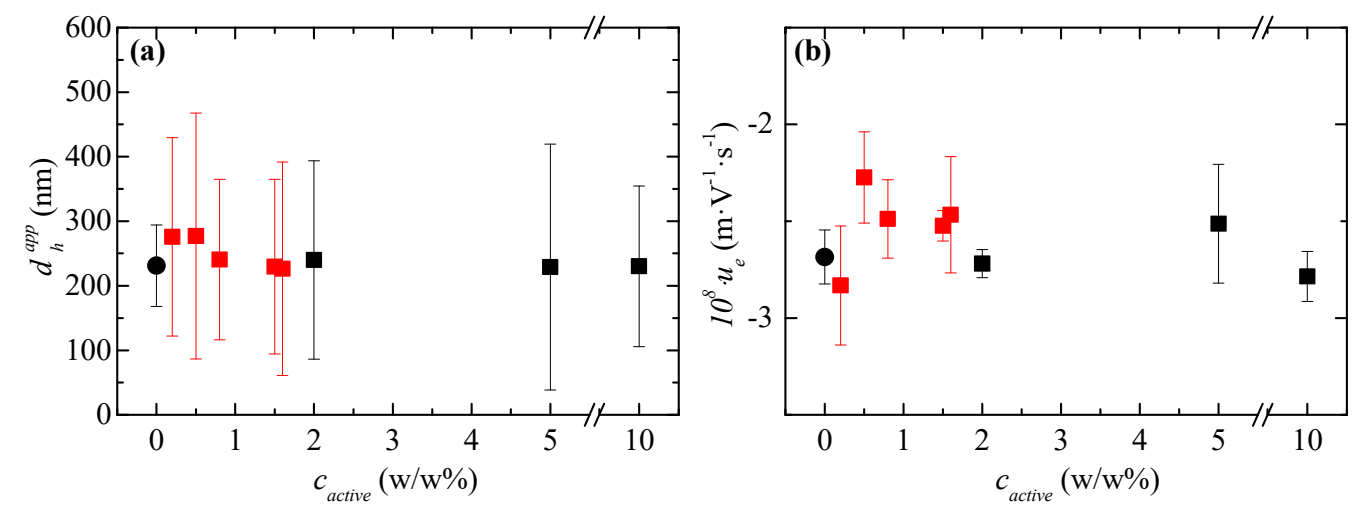

Figure 5. (a) Dependence of the apparent hydrodynamic diameter on the encapsulated active ingredient concentration for nanoemulsions with an oil:surfactant ratio of 1 and a fixed amount of water of $75 \mathrm{w} / \mathrm{w} \%$. The error bars provide information of the width at half height of the hydrodynamic diameter distributions. (b) Dependence of the electrophoretic mobility on the encapsulated active ingredients' concentration for a nanoemulsion with an oil:surfactant ratio of 1 and a fixed amount of water of $75 \mathrm{w} / \mathrm{w} \%$. In the both panels: $(\bullet)$ nanoemulsions without active ingredient, ( $\square)$ nanoemulsions with encapsulated ceramide, and (ם) nanoemulsions with minoxidil. Notice that the high turbidity of the mixtures makes necessary a dilution of the sample by a factor 10 for performing DLS measurements.

Despite the maximum amount of encapsulated minoxidil is several times higher than that of ceramide, there are no changes neither on the droplet size nor on the electrophoretic mobility with the concentration of minoxidil, remaining both parameters very close to those obtained for nanoemulsions containing ceramide (see Table 3 for a summary of the effect of the inclusion of minoxidil on the size and surface charge of the droplets). In particular, the absence of any change on the electrophoretic mobility with the minoxidil concentration suggests that, on the contrary to that what happens for the encapsulation of the ceramide-like molecule, minoxidil molecules are placed only in the inner region of the oil core of the droplets with a very limited incorporation at the droplet/aqueous continuous phase interface, and hence the effective charge of the droplets loaded with minoxidil continues to be defined by the dissociated groups of the oleic acid placed in the protecting shell of the droplets. This may be understood easily considering that CER is a clear amphiphilic molecule, whereas the amphiphilicity of minoxidil is lower and hence its adsorption at the fluid/fluid interface is rather limited (see Figure 1).

Table 3. Average hydrodynamic diameter, polydispersity index, electrophoretic mobility and zeta potential for nanoemulsions with a water content of $75 \mathrm{w} / \mathrm{w} \%$ and a fixed oil ratio of 1 loaded with different amounts of the minoxidil.

\begin{tabular}{|c|c|c|c|c|}
\hline $\begin{array}{c}\text { Loaded CER } \\
(w / w \%)\end{array}$ & $\begin{array}{l}d_{h}^{a p p} \\
(\mathrm{~nm})\end{array}$ & PDI & $\begin{array}{c}10^{8} u_{e} \\
\left(\mathrm{~m} \cdot \mathrm{V}^{-1} \cdot \mathrm{s}^{-1}\right)\end{array}$ & $\begin{array}{c}\text { Zeta Potential } \\
(\mathrm{mV})\end{array}$ \\
\hline 0 & 231 & 0.137 & $-(2.7 \pm 0.1)$ & $-(30 \pm 2)$ \\
\hline 2 & 240 & 0.282 & $-(2.7 \pm 0.1)$ & $-(31+2)$ \\
\hline 5 & 229 & 0.286 & $-(2.5 \pm 0.3)$ & $-(30 \pm 5)$ \\
\hline 10 & 230 & 0.272 & $-(2.8 \pm 0.1)$ & $-(31 \pm 3)$ \\
\hline
\end{tabular}

To further increase the maximum amount of encapsulated ceramide-like molecule, nanoemulsions with different compositions were tested. Table 4 summarized the maximum amount of ceramide-like molecule encapsulated in nanoemulsions as function of the water content at a fixed oil:surfactant ratio of 1, i.e., in nanoemulsions containing different volumes of oil phase and hence different numbers of droplets for solubilizing the ceramidelike molecule. Thus, the lower the water content the higher the amount of oil contained within the formulation. 
Table 4. Maximum amount of ceramide-like molecule that can be included without any signature of sedimentation as function of the composition of the formulations.

\begin{tabular}{ccc}
\hline $\begin{array}{c}\text { Water } \\
\mathbf{( w / w \% )}\end{array}$ & Ratio Oleic Acid/Tween 80 & $\begin{array}{c}\text { Maximum Amount of Encapsulated CER } \\
\mathbf{( w / w} \%)\end{array}$ \\
\hline 73.00 & 1.00 & 2.00 \\
75.00 & 1.00 & 1.60 \\
77.00 & 1.00 & 1.50 \\
80.00 & 1.00 & 1.50 \\
\hline
\end{tabular}

The results show that the increase in the amount of oil phase results in an enhancement of the encapsulation of the ceramide-like molecule, which may be ascribed to the higher volume available for its distribution. Thus, considering that the increase in the oil content of the nanoemulsions accompanied of an equivalent increase in the surfactant leads only to an increase in the number of droplets, it may be expected that more oily nanoemulsions can favor the solubilization of higher concentrations of actives. On the other hand, the increase in the water content above $75 \mathrm{w} / \mathrm{w} \%$ leads to a slight decrease in the maximum amount of ceramide that can be incorporated within the nanoemulsion without compromising its stability.

\subsection{Long-Term Stability of Nanoemulsions Loaded with CER and MIN}

The long-term stability of the dispersions containing the hydrophobic actives can be followed in terms of the time evolution of apparent hydrodynamic diameter of the droplets. Figure 6 shows the time evolution of the apparent hydrodynamic diameter of the droplets of nanoemulsions with a water content of $75 \mathrm{w} / \mathrm{w} \%$ and a fixed oilssurfactant ratio of 1 loaded with different concentration of the hydrophobic actives.
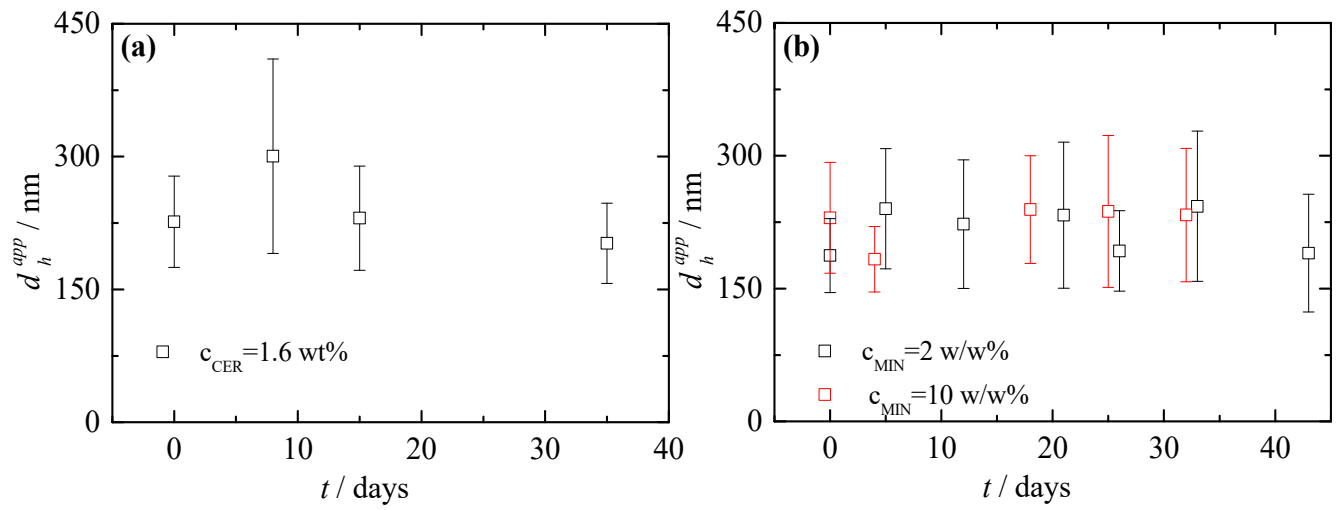

Figure 6. Dependence of the apparent hydrodynamic diameter on the aging time at room temperature for nanoemulsions with an oil: surfactant ratio of 1 and a fixed amount of water of $75 \mathrm{w} / \mathrm{w} \%$ loaded with different amount of CER (a) and MIN (b). The error bars provide information of the width at half height of the hydrodynamic diameter distributions.

The temporal evolution of the apparent hydrodynamic diameter of the nanoemulsions loaded with CER and MIN appears rather constant during the first month of aging. Thus, it is possible to assume that the storage of the nanoemulsions loaded with the actives does not undergo any significant destabilization upon their storage at room temperature. This makes it possible to assume that the studied nanoemulsions can present good long-term stability, which is further confirmed by the absence of any creaming or sedimentation on the dispersion during a period longer of one year. 


\section{Conclusions}

Different oil-in-water nanoemulsions with relatively simple compositions were tested as platforms for the encapsulation of two molecules of potential interest in the cosmetics industry: a ceramide-like molecule and minoxidil. The results show that these two active ingredients can be efficiently encapsulated using nanoemulsions containing a water fraction in the range $68-80 \mathrm{w} / \mathrm{w} \%$, and a very reduced amount of oil (in the range $10-16 \mathrm{w} / \mathrm{w} \%$ ), maintaining a ratio between the oil content and that of the stabilizing agent (Tween 80) of 1 . This makes possible the preparation of formulations loading up to a maximum of $2 \mathrm{w} / \mathrm{w} \%$ of the ceramide-like molecule for nanoemulsions having a water content of $73 \mathrm{w} / \mathrm{w} \%$ and an oil:surfactant ratio of 1 , and more than $10 \mathrm{w} / \mathrm{w} \%$ of minoxidil, within oleic acid droplets having an average size about $200 \mathrm{~nm}$. Despite the fact that the content of oil that can be loaded into the droplets can be modulated by changing the composition of the formulation, this affects neither to the size nor the average surface charge of the droplets. This is explained considering that the only effect of the modification of the amount of the oil and aqueous phases maintaining a constant oil:surfactant ratio is to change the number of droplets and hence the accessible volume for the distribution of the actives. The nanoemulsions' droplets remain stable due to their effective negative charge. It is true that this work has been only focused on the optimization of the encapsulation efficiency of nanoemulsions for a ceramidelike molecule and minoxidil. However, the results suggest that this type of colloidal dispersion may provide a versatile tool for enhancing the distribution of hydrophobic actives with cosmetic interest. This is possible because the methodology used in this work allows the preparation of stable formulations taking advantage of the ability of a non-ionic surfactant for changing its properties due to a temperature change, without needing any organic solvent or co-surfactant for ensuring the dispersion of the active substance.

Author Contributions: Conceptualization, E.G.; methodology, E.G., L.F.-P., L.R. and M.B.; software, E.G. and L.F.-P.; validation, E.G., F.O. and R.G.R.; formal analysis, E.G. and L.F.-P.; investigation, E.G., L.F.-P., L.R., M.B., F.O. and R.G.R.; resources, F.O. and R.G.R.; data curation, E.G. and L.F.-P.; writingoriginal draft preparation, E.G., L.F.-P., L.R. and M.B.; writing-review and editing, E.G., F.O. and R.G.R.; visualization, E.G. and L.F.-P.; supervision, E.G., L.F.-P. and R.G.R.; project administration, E.G., F.O. and R.G.R.; funding acquisition, E.G., F.O. and R.G.R. All authors have read and agreed to the published version of the manuscript.

Funding: This work was funded by MICINN under grant PID2019-106557GB-C21, by Banco Santander-Universidad Complutense grant PR87/19-22513 (Spain) and by the E.U. via the framework of the European Innovative Training Network-Marie Sklodowska-Curie Action NanoPaint (grant agreement 955612).

Institutional Review Board Statement: Not applicable.

Informed Consent Statement: Not applicable.

Data Availability Statement: Data are available upon request.

Conflicts of Interest: The authors declare no conflict of interest.

\section{References}

1. Lu, W.; Kelly, A.L.; Miao, S. Emulsion-based encapsulation and delivery systems for polyphenols. Trends Food Sci. Technol. 2016, 47, 1-9. [CrossRef]

2. Guimarães, K.L.; Ré, M.I. Lipid Nanoparticles as Carriers for Cosmetic Ingredients: The First (SLN) and the Second Generation (NLC). In Nanocosmetics and Nanomedicines: New Approaches for Skin Care; Beck, R., Guterres, S., Pohlmann, A., Eds.; Springer: Berlin/Heidelberg, Germany, 2011; pp. 101-122.

3. Lucia, A.; Argudo, P.G.; Guzmán, E.; Rubio, R.G.; Ortega, F. Formation of surfactant free microemulsions in the ternary system water/eugenol/ethanol. Colloids Surf. A 2017, 521, 133-140. [CrossRef]

4. Argudo, P.G.; Guzmán, E.; Lucia, A.; Rubio, R.G.; Ortega, F. Preparation and Application in Drug Storage and Delivery of Agarose Nanoparticles. Int. J. Polym. Sci. 2018, 2018, 7823587. [CrossRef] 
5. Cáceres, M.; Guzmán, E.; Alvarez-Costa, A.; Ortega, F.; Rubio, R.G.; Coviella, C.; Santo Orihuela, P.L.; Vassena, C.V.; Lucia, A. Surfactantless Emulsions Containing Eugenol for Imidacloprid Solubilization: Physicochemical Characterization and Toxicity against Insecticide-Resistant Cimex lectularius. Molecules 2020, 25, 2290. [CrossRef]

6. Lucia, A.; Guzmán, E.; Rubio, R.G.; Ortega, F. Enhanced solubilization of an insect juvenile hormone (JH) mimetic (piryproxyfen) using eugenol in water nanoemulsions stabilized by a triblock copolymer of poly(ethylenglycol) and poly(propilenglycol). Colloids Surf. A 2020, 606, 125513. [CrossRef]

7. Lucia, A.; Guzmán, E. Emulsions containing essential oils, their components or volatile semiochemicals as promising tools for insect pest and pathogen management. Adv. Colloid Interface Sci. 2021, 287, 102330. [CrossRef]

8. Mateos-Maroto, A.; Abelenda-Núñez, I.; Ortega, F.; Rubio, R.G.; Guzmán, E. Polyelectrolyte Multilayers on Soft Colloidal Nanosurfaces: A New Life for the Layer-By-Layer Method. Polymers 2021, 13, 1221. [CrossRef]

9. Vieira, M.V.; Pastrana, L.M.; Fuciños, P. Microalgae Encapsulation Systems for Food, Pharmaceutical and Cosmetics Applications. Mar. Drugs 2020, 18, 644. [CrossRef]

10. Kuang, S.S.; Oliveira, J.C.; Crean, A.M. Microencapsulation as a Tool for Incorporating Bioactive Ingredients into Food. Crit. Rev. Food Sci. Nutr. 2010, 50, 951-968. [CrossRef]

11. Ashaolu, T.J. Nanoemulsions for health, food, and cosmetics: A review. Environ. Chem. Lett. 2021. [CrossRef] [PubMed]

12. McClements, D.J. Emulsion Design to Improve the Delivery of Functional Lipophilic Components. Ann. Rev. Food Sci. Technol. 2010, 1, 241-269. [CrossRef]

13. Fernández-Peña, L.; Gutiérrez-Muro, S.; Guzmán, E.; Lucia, A.; Ortega, F.; Rubio, R.G. Oil-In-Water Microemulsions for Thymol Solubilization. Colloids Interfaces 2019, 3, 64. [CrossRef]

14. Spada, F.; Barnes, T.M.; Greive, K.A. Skin hydration is significantly increased by a cream formulated to mimic the skin's own natural moisturizing systems. Clin. Cosmet. Investig. Dermatol. 2018, 11, 491-497. [CrossRef]

15. Simonazzi, A.; Cid, A.G.; Villegas, M.; Romero, A.I.; Palma, S.D.; Bermúdez, J.M. Nanotechnology applications in drug controlled release. In Drug Targeting and Stimuli Sensitive Drug Delivery Systems; Grumezescu, A.M., Ed.; William Andrew Publishing: Norwich, NY, USA, 2018; pp. 81-116. [CrossRef]

16. Venkataramani, D.; Tsulaia, A.; Amin, S. Fundamentals and applications of particle stabilized emulsions in cosmetic formulations. Adv. Colloid Interface Sci. 2020, 283, 102234. [CrossRef]

17. Patzelt, A.; Richter, H.; Dähne, L.; Walden, P.; Wiesmüller, K.-H.; Wank, U.; Sterry, W.; Lademann, J. Influence of the Vehicle on the Penetration of Particles into Hair Follicles. Pharmaceutics 2011, 3, 307-314. [CrossRef]

18. Sun, R.; Xia, N.; Xia, Q. Non-aqueous nanoemulsions as a new strategy for topical application of astaxanthin. J. Dispers. Sci. Technol. 2020, 41, 1777-1788. [CrossRef]

19. Kahraman, E.; Kaykın, M.; Şahin Bektay, H.; Güngör, S. Recent Advances on Topical Application of Ceramides to Restore Barrier Function of Skin. Cosmetics 2019, 6, 52. [CrossRef]

20. Goren, A.; Shapiro, J.; Roberts, J.; McCoy, J.; Desai, N.; Zarrab, Z.; Pietrzak, A.; Lotti, T. Clinical utility and validity of minoxidil response testing in androgenetic alopecia. Dermatol. Ther. 2015, 28, 13-16. [CrossRef] [PubMed]

21. Dawber, R.P.; Rundegren, J. Hypertrichosis in females applying minoxidil topical solution and in normal controls. J. Eur. Acad. Dermatol. Venereol. JEADV 2003, 17, 271-275. [CrossRef] [PubMed]

22. Santos, A.C.; Pereira-Silva, M.; Guerra, C.; Costa, D.; Peixoto, D.; Pereira, I.; Pita, I.; Ribeiro, A.J.; Veiga, F. Topical MinoxidilLoaded Nanotechnology Strategies for Alopecia. Cosmetics 2020, 7, 21. [CrossRef]

23. Naseema, A.; Kovooru, L.; Behera, A.K.; Kumar, K.P.P.; Srivastava, P. A critical review of synthesis procedures, applications and future potential of nanoemulsions. Adv. Colloid Interface Sci. 2021, 287, 102318. [CrossRef]

24. Tong, K.; Zhao, C.; Sun, Z.; Sun, D. Formation of Concentrated Nanoemulsion by W/O Microemulsion Dilution Method: Biodiesel, Tween 80, and Water System. ACS Sustain. Chem. Eng. 2015, 3, 3299-3306. [CrossRef]

25. Shinoda, K.; Saito, H. The Stability of O/W type emulsions as functions of temperature and the HLB of emulsifiers: The emulsification by PIT-method. J. Colloid Interface Sci. 1969, 30, 258-263. [CrossRef]

26. Shinoda, K.; Saito, H. The effect of temperature on the phase equilibria and the types of dispersions of the ternary system composed of water, cyclohexane, and nonionic surfactant. J. Colloid Interface Sci. 1968, 26, 70-74. [CrossRef]

27. Llamas, S.; Guzmán, E.; Baghdadli, N.; Ortega, F.; Cazeneuve, C.; Rubio, R.G.; Luengo, G.S. Adsorption of poly (diallyldimethylammonium chloride)—sodium methyl-cocoyl-taurate complexes onto solid surfaces. Colloids Surf. A 2016, 505, 150-157. [CrossRef]

28. Guzmán, E.; Llamas, S.; Fernández-Peña, L.; Léonforte, F.; Baghdadli, N.; Cazeneuve, C.; Ortega, F.; Rubio, R.G.; Luengo, G.S. Effect of a natural amphoteric surfactant in the bulk and adsorption behavior of polyelectrolyte-surfactant mixtures. Colloids Surf. A 2020, 585, 124178. [CrossRef]

29. Hernández-Rivas, M.; Guzmán, E.; Fernández-Peña, L.; Akanno, A.; Greaves, A.; Léonforte, F.; Ortega, F.; Rubio, R.G.; Luengo, G.S. Deposition of Synthetic and Bio-Based Polycations onto Negatively Charged Solid Surfaces: Effect of the Polymer Cationicity, Ionic Strength, and the Addition of an Anionic Surfactant. Colloids Interfaces 2020, 4, 33. [CrossRef]

30. Mateos, H.; Valentini, A.; Robles, E.; Brooker, A.; Cioffi, N.; Palazzo, G. Measurement of the zeta-potential of solid surfaces through Laser Doppler Electrophoresis of colloid tracer in a dip-cell: Survey of the effect of ionic strength, $\mathrm{pH}$, tracer chemical nature and size. Colloids Surf. A 2019, 576, 82-90. [CrossRef] 
31. Mateos, H.; Valentini, A.; Lopez, F.; Palazzo, G. Surfactant Interactions with Protein-Coated Surfaces: Comparison between Colloidal and Macroscopically Flat Surfaces. Biomimetics 2020, 5, 31. [CrossRef] [PubMed]

32. Mateos, H.; Valentini, A.; Colafemmina, G.; Murgia, S.; Robles, E.; Brooker, A.; Palazzo, G. Binding isotherms of surfactants used in detergent formulations to bovine serum albumin. Colloids Surf. A 2020, 598, 124801. [CrossRef]

33. Lucia, A.; Toloza, A.C.; Fanucce, M.; Fernández-Peña, L.; Ortega, F.; Rubio, R.G.; Coviella, C.; Guzmán, E. Nanoemulsions based on thymol-eugenol mixtures: Characterization, stability and larvicidal activity against Aedes aegypti. Bull. Insectol. 2020, 73, 153-160.

34. Hunter, R.J. Zeta Potential in Colloid Science: Principles and Applications; Academic Press: London, UK, 1998.

35. Smoluchowski, M. Handbuch der Electrizität und des Magnetismus (Graetz); Barth: Leipzig, Germany, 1921.

36. Berne, B.J.; Pecora, R. Dynamic Light Scattering: With Applications to Chemistry, Biology, and Physics; Dover Publications Inc.: Mineola, NY, USA, 2003.

37. Exerowa, D.; Zacharieva, M.; Cohen, R.; Platikanov, D. Dependence of the equilibrium thickness and double layer potential of foam films on the surfactant concentration. Colloids Polym. Sci. 1979, 257, 1089-1098. [CrossRef]

38. Roldan-Cruz, C.; Vernon-Carter, E.J.; Alvarez-Ramirez, J. Assessing the stability of Tween 80-based O/W emulsions with cyclic voltammetry and electrical impedance spectroscopy. Colloids Surf. A 2016, 511, 145-152. [CrossRef]

39. Ghonemy, S.; Alarawi, A.; Bessar, H. Efficacy and safety of a new 10\% topical minoxidil versus $5 \%$ topical minoxidil and placebo in the treatment of male androgenetic alopecia: A trichoscopic evaluation. J. Dermatol. Treat. 2021, 32, 236-241. [CrossRef] [PubMed] 